\title{
Health-related quality of life and medication adherence in elderly patients with epilepsy
}

\author{
Soudabeh Hamedi-Shahraki ${ }^{1}$, Mohammad-Reza Eshraghian ${ }^{1}$, Mir-Saeed Yekaninejad ${ }^{1}$, \\ Mehdi Nikoobakht ${ }^{2}$, Aliakbar Rasekhi ${ }^{3}$, Hui Chen ${ }^{4}$, Amir Pakpour ${ }^{5}$ \\ ${ }^{1}$ Department of Epidemiology and Biostatistics, School of Public Health, Tehran University of Medical Sciences, Tehran, Iran \\ ${ }^{2}$ Department of Neurosurgery, Iran University of Medical Sciences, Tehran, Iran \\ ${ }^{3}$ Department of Biostatistics, Faculty of Medical Sciences, Tarbiat Modares University, Tehran, Iran \\ ${ }^{4}$ School of Life Sciences, Faculty of Science, University of Technology Sydney, Sydney, Australia
}

${ }^{5}$ Social Determinants of Health Research Centre, Qazvin University of Medical Sciences, Shahid Bahounar BLV, 3419759811, Qazvin, Iran;

Department of Nursing, School of Health and Welfare, Jönköping University, Jönköping, Sweden

\section{ABSTRACT}

Objective. Considering the high prevalence of epilepsy in the elderly and the importance of maximising their quality of life $(\mathrm{Q} \mathrm{L})$, this study aimed to investigate the relationship between medication adherence and QoL, and the mediating effects of medication adherence on the association between serum antiepileptic drug (AED) level and seizure severity with QoL in elderly epileptics.

Methods. In a longitudinal study, 766 elderly patients with epilepsy who were prescribed a minimum of one antiepileptic drug were selected by convenience sampling method. A Medication Adherence Report Scale (MARS-5) questionnaire was completed at the baseline. Seizure severity and QoL were assessed after six months using the Liverpool Seizure Severity Scale (LSSS) and the QoL in Epilepsy (QOLIE-31) questionnaires respectively. Serum level of AED was also measured at six-month follow-up. Results. Medication adherence was significantly correlated with both seizure severity $(\beta=-0.33, p<0.0001)$ and serum AED level $(\beta=0.29, p<0.0001)$ after adjusting for demographic and clinical characteristics. Neither QoL nor its sub-classes were correlated with seizure severity. In addition, a significant correlation was not observed between serum AED level and QoL. However, medication adherence was significantly correlated with $\mathrm{QoL}(\beta=0.30, p<0.0001)$. The mediating effects of medication adherence on the association between serum AED level $(Z=3.39, p<0.001)$ and seizure severity $(Z=-3.47, p<0.001)$ with QoL were supported by the Sobel test.

Conclusion. This study demonstrates that medication adherence has a beneficial impact on QoL in elderly epileptics. Therefore, adherence to treatment should be monitored to improve their QoL.

Key words: epilepsy, medication adherence, seizure, quality of life, elderly

(Neurol Neurochir Pol 2019; 53 (2): 123-130)

\section{Introduction}

Epilepsy, a neurological disorder characterised by recurrent unprovoked seizures, is one of the most common chronic brain disorders globally [1]. Despite advances in the understanding of the pathophysiological mechanisms of epilepsy and the development of medical treatments in recent decades, people of all ages with this neurological disorder continue to

Address for correspondence: Mir-Saeed Yekaninejad, Department of Epidemiology and Biostatistics, Tehran University of Medical Sciences, Tehran, Iran, e-mail: yekaninejad@sina.tums.ac.ir 
be stigmatised by it [2]. Epilepsy is one of the world's longest-recognised medical conditions; it affects approximately 50 million people worldwide with nearly $80 \%$ of them living in developing countries [3]. The prevalence of epilepsy in the Iranian population is between $7.8-18 / 1,000$ persons or approximately $1.4 \%$ of the population $[4,5]$, which is slightly higher than the reported prevalence of $5.8-8.4 / 1,000$ persons in developed countries $[1,6]$. This disease affects all ages, ethnicities and socioeconomic groups; however, its prevalence and incidence increase markedly in individuals over the age of 65 years, even more than they do in infancy, another high prevalence age. In fact epilepsy is the third most common neurological disease in the elderly, after stroke and dementia $[7,8]$. Taking into account the future growth of the world's population aged $>60$ years [9] due to increasing life expectancy, the prevalence of epilepsy is predicted to dramatically rise over the next few decades.

Medication adherence is a fundamental determinant of effective treatment [10] that is defined by the World Health Organisation as "the degree to which the person's behaviour corresponds to the agreed recommendations from a health care provider" [11]. Epilepsy has been successfully controlled with medication adherence, and most people with epilepsy (almost 70\% of patients) can become seizure-free by taking one anti-seizure medication daily, called an anti-epileptic drug (AED) $[12,13]$. Nevertheless, non-adherence to medication is a very common phenomenon in patients with chronic diseases [14-18] including epilepsy [18-23], which not only affects the individual's health but also the healthcare system. This leads to uncontrolled symptoms and substantial deleterious effects on the quality of life (QoL) and those patients can become a heavy burden on society and healthcare systems [24-26].

Elderly people with epilepsy generally respond well to AED treatment. Up to $80 \%$ of patients with late-onset epilepsy can be expected to remain seizure-free with AED treatment [27]; however, only $38-57 \%$ (average $<45 \%$ ) of elderly epileptic patients have good adherence to AEDs [28]. Explanations for poor adherence in this population of patients include patient-physician discordance, complex medication regimen, the frequency of administration of multiple medications, disturbances of memory, specific beliefs about drugs, being depressed or anxious, and unusual times during the day to take the medication [25]. Although patient understanding of the optimal care, and improving their QoL, are problematic in epileptic treatment, it remains questionable whether adherence to AED can actually improve QoL in this population. The impact of AED therapy on the QoL in younger groups of patients has been previously reported $[10,29,30]$ and positive and negative effects of AED therapy on QoL have been demonstrated [31]. However, the impact of AED treatment in terms of QoL among the elderly has not been well investigated.

Therefore, the primary aim of this study was to assess the QoL and its correlation with AED adherence among elderly patients with epilepsy. In addition, we aimed to evaluate the mediating effects of medication adherence in the relationship between serum AED level and seizure severity with QoL. To obtain more accurate information on AED adherence, both a self-reporting method (Medication Adherence Report Scale, MARS-5) and serum AED level measurement were used. The correlation between MARS- 5 score and serum AED level and whether MARS- 5 score can be replaced with serum AED level to predict the QoL in the elderly with epilepsy was also examined.

\section{Material and methods}

\section{Study design and participants}

This longitudinal study was carried out on 766 elderly patients with epilepsy referred to six neurology clinics between March 2014 and December 2015. The patients were recruited through convenience sampling. The subjects included males and females aged 65 years or above with a confirmed diagnosis of epilepsy according to the International League Against Epilepsy Criteria [32]. The subjects had the ability to perform daily activities, had been prescribed at least two AEDs, and had no major cognitive impairment (defined as a score of 23 or below on the Mental State Examination, MSE) or an acute psychiatric disorder diagnosed by a psychiatrist. Exclusion criteria were a history of drug abuse, diabetes, cardiovascular diseases, rheumatoid arthritis, malignant tumours, kidney dysfunctions and liver diseases, being in receipt of medications other than AEDs at the time of recruitment or throughout the follow-up period, and being unwilling to participate in the study.

All applicants were screened by a trained physician for eligibility according to these inclusion and exclusion criteria. All eligible participants were given a full explanation of the objectives and protocol of the study, and written informed consent was signed prior to enrolment. Demographic characteristics, clinical features and medication adherence were then collected from the participants. Six months later, the subjects were invited to attend the clinic to complete the questionnaires evaluating seizure severity and QoL. Blood samples were taken on the same day for the measurement of AED level as an objective assessment of AED adherence.

\section{Instruments \\ Background information sheet and medical records}

Demographic characteristics including age, sex, marital status, educational years, employment and monthly income $($ High $>\$ 1,000 ;$ Intermediate $=\$ 500-1,000 ;$ Low $<\$ 500)$ were collected by face-to-face interviews and recorded in the Background Information Sheet. The clinical features of the participants, including the type of epilepsy and the duration of the disease, were also collected from their medical records. 


\section{Medication Adherence Report Scale (MARS-5)}

MARS-5 is a self-reporting and widely applicable questionnaire for the subjective assessment of medication adherence [33]. It consists of five statements of non-adherent behaviours (I forgot to take my antiepileptic medicine, I altered the dose of my antiepileptic medicine, I stopped taking my antiepileptic medicine for a while, I decided to miss a dose of my antiepileptic medicine, I took less antiepileptic medicines than prescribed) answered on a five-point Likert scale ( 1 = always, 2 = often, $3=$ sometimes, $4=$ rarely, $5=$ never), giving an overall score that ranges between 5 and 25. According to the threshold, a MARS- 5 score of 20 or above is considered as high adherence [14]. The concurrent validity and internal consistency of the MARS- 5 questionnaire has been supported by previous studies [34]. The participants completed the MARS-5 questionnaire at the baseline.

\section{Liverpool Seizure Severity Scale (LSSS)}

The participants were asked to complete the LSSS questionnaire if they reported more than one week had elapsed since their last seizure. The LSSS questionnaire consists of 20 items rated on a Likert scale. A four-point Likert scale was used to respond to each item, with higher points indicating greater seizure severity. It has been demonstrated that LSSS is a valid and reliable instrument quantifying seizure severity that may also be used to evaluate changes in seizure severity over time [35]. Furthermore, the known-group validity showed subjects with severe seizure symptoms could be distinguished from those with minor seizure symptoms by LSSS [35].

\section{Serum AED level}

In order to measure serum concentrations at the six-month follow-up of the three most commonly used AEDs i.e. phenytoin, lamotrigine and carbamazepine, whole blood samples were taken prior to the next daily routine dose of drug. The serum was then separated, and AED concentrations were measured using a microparticle enzyme immunostimulatory assay kit (Abbott Axsym ${ }^{\circledR}$, Abbott Laboratories, Abbott Park, IL, USA). The therapeutic dose range of AED which can prevent seizures effectively without toxic effects has been reported in detail [36]. The serum concentrations of AEDs were categorised into three groups based on the reference as 'below the therapeutic range', 'within the therapeutic range', and 'above the therapeutic range'.

\section{Quality of Life in Epilepsy (QOLIE-31)}

QoL was evaluated using a QOLIE-31 questionnaire, which was designed exclusively to assess an epileptic patient's QoL at six-month follow-up. It consists of 31 items in seven subclasses: seizure concerns, cognitive function, energy/ fatigue, emotional wellbeing, social function, medication efficacy, and overall QoL [37]. The subscale scores range from 0 to 100 points, and the higher the score, the better the QoL.
The overall score of QOLIE-31 can be calculated by weighting and summarising seven-dimension scores. The Persian version of QOLIE-31 has been shown to be a reliable instrument for assessing QoL in patients with epilepsy [38].

\section{Statistical analysis}

The results were expressed as mean \pm standard deviation (SD) for quantitative data with normal distribution, and frequency (percent) for qualitative data. The analyses were performed in several steps. Firstly, Pearson correlation analysis was used to investigate the relationships between serum AED level, MARS- 5 score, LSSS score, and overall QOLIE-31score. The relationships between serum AED level and LSSS score with the MARS-5 score were then evaluated by linear regression after adjusting for potential confounders (i.e. age, sex, marital status, education years, employment status, monthly income, type of epilepsy and disease duration). The relationships between independent variables (serum AED level, LSSS and MARS-5 score) and dependent variable (each sub-class of QoL and the overall score of the QoL) were also evaluated using multiple linear regression analysis, after controlling for potential confounders. Based on the Bonferroni correction, $p<0.00625(0.05 / 8)$ is considered as significant for multiple comparisons between the eight regression models.

Lastly, structural equation modelling (SEM) was used to test the model and investigate whether seizure severity and serum AED level directly correlated with the QoL, or whether it was being mediated through other factors. Several fit indices, including chi-square statistics, Comparative Fit Index (CFI), Goodness-of-Fit Index (GFI), Tucker-Lewis Index (TLI), Normed Fit Index (NFI) and Root Mean Square Error of Approximation (RMSEA), were evaluated to determine the model fit. To interpret these indices, the following criteria were used: $\chi^{2} / \mathrm{df}$ ratio $<2$ (excellent); $\chi^{2} / \mathrm{df}<3$ (good); $\chi^{2} / \mathrm{df}<5$ (acceptable); $\geq 0.90$ as good fit for CFI, GFI, TLI, and NFI; $\leq 0.08$ as good fit for RMSEA[39]. Sobel test was also used to examine the significance of mediation effects. The descriptive analyses and regression models were performed using IBM SPSS version 21.0 software; SEM was conducted using AMOS 21.

\section{Results}

The demographic and clinical characteristics of the participants are set out in Table 1 . The average age of participants was $73.9 \pm 5.7$ years, and more than half were female (54.7\%). Most participants were married and had low levels of education. All patients received polytherapy with a minimum of two AEDs. Therefore, it is not possible to assess the effect of each type of AED on adherence. As shown in Table 1, more than two-thirds of participants had focal epilepsy (70.2\%). Of the patients with focal epilepsy, 97 (18\%) reported focal seizures without impairment of consciousness, and $68(13 \%)$ patients reported focal seizures with impairment of consciousness. 
All patients with generalised epilepsy reported convulsive seizures.

The mean overall score of the QOLIE-31 among the participants was $67.8 \pm 20.5$, with energy/fatigue the worst domain (59.3 \pm 18.6$)$ and medication efficacy the best domain (78.7 \pm 24.1$)$ (Tab. 1). Furthermore, nearly half of the participants (48.7\%) had serum AED levels below the therapeutic range (i.e. categorised as non-adherent).

In the first step, we investigated the relationship between the variables. All of the correlation coefficients were statistically significant. In particular, MARS-5 score was positively correlated with serum AED level $(\mathrm{r}=0.36, p<0.001)$, and inversely correlated with LSSS score $(r=-0.39, p<0.001)$. In addition, a positive correlation was observed between QOLIE-31 score and MARS-5 score $(r=0.33, p<0.001)$.

The relationships between the variables were then evaluated by multiple linear regression after adjusting for potential confounders. Table 2 shows the results from the regression models that evaluated the association between MARS- 5 score with LSSS score and serum AED level. As shown in Table 2, MARS-5 score was significantly correlated with both LSSS $(\beta=-0.33, p<0.0001)$ and serum AED level $(\beta=0.29$, $p<0.0001)$ after adjusting for confounders. In other words, LSSS and serum AED level were significant predictors of MARS- 5 score and accounted for almost a quarter of the variation in the MARS- 5 score.

The relationships between LSSS score, MARS- 5 score and serum AED level with overall QOLIE-31 score and its domains are set out in Table 3. Neither overall QOLIE-31 score nor its sub-classes were correlated with LSSS score. Similarly, no significant correlation was observed between serum AED level and overall QOLIE-31 score and the scores in QOLIE-31 domains after adjusting for demographic and clinical confounders. However, MARS-5 score was significantly correlated with the overall score of QOLIE-31 $(\beta=0.30, p<0.0001)$ and its domains.

After confirming the correlation between MARS- 5 score and QOLIE-31 score, we tried to examine the proxy effects of MARS- 5 on QoL using the SEM. The SEM analysis is graphically described in Figure 1. This model showed an acceptable fit. According to SEM analysis, all the goodness-of-fit indices indicated an acceptable fit, except for the $\chi^{2}$ which was statistically significant $(p<0.001)$. However, the value of the $\chi^{2} / \mathrm{df}$ ratio was good $(3.105<5)$. The rest of the fit indices showed an acceptable fit, with CFI, GFI, TLI and NFI above 0.9 , and RMSEA less than $0.08(\mathrm{CFI}=0.974, \mathrm{GFI}=0.978, \mathrm{TLI}=0.957$, $\mathrm{NFI}=0.963$, RMSEA $=0.052$ ). The standardised coefficients of LSSS score on QOLIE-31 score (-0.015) and serum AED level on QOLIE-31 (0.021) were not significant, while the standardised coefficients of LSSS score on MARS- 5 score $(-0.316)$ and serum AED level on MARS-5 score (0.268) were significant. Furthermore, the mediating effects of MARS- 5 score was investigated using the Sobel test. According to the Sobel test, LSSS $(\mathrm{Z}=-3.47, p<0.001)$ and the serum AED level $(\mathrm{Z}=3.39$,
Table 1. Demographic and clinical characteristics of the participants $(n=766)$

\begin{tabular}{|c|c|}
\hline Age (years) & $73.94 \pm 5.77$ \\
\hline Male, n (\%) & $347(45.3 \%)$ \\
\hline Married, n (\%) & $582(76.0 \%)$ \\
\hline Education (years) & $8.91 \pm 5.10$ \\
\hline Employed, n (\%) & $276(36.0 \%)$ \\
\hline \multicolumn{2}{|l|}{ Monthly income, n (\%) } \\
\hline High $(>\$ 1,000)$ & $162(21.2 \%)$ \\
\hline Intermediate $(\$ 500-1,000)$ & $461(60.2 \%)$ \\
\hline $\operatorname{Low}(<\$ 500)$ & $143(18.7 \%)$ \\
\hline \multicolumn{2}{|l|}{ Epilepsy type, n (\%) } \\
\hline Generalised & $228(29.8 \%)$ \\
\hline Focal & $538(70.2 \%)$ \\
\hline \multicolumn{2}{|l|}{ Aetiology, n (\%) } \\
\hline Vascular & $214(28.0 \%)$ \\
\hline Trauma & $122(15.9 \%)$ \\
\hline Idiopathic/cryptogenic & $430(56.1 \%)$ \\
\hline \multicolumn{2}{|l|}{ Type of medication, $n$ (\%) } \\
\hline Phenytoin & $506(66.0 \%)$ \\
\hline Lamotrigine & $333(43.5 \%)$ \\
\hline Carbamazepine & $305(39.8 \%)$ \\
\hline Oxcarbazepine & $204(26.6 \%)$ \\
\hline Phenobarbital & $148(19.3 \%)$ \\
\hline Topiramate & $73(9.5 \%)$ \\
\hline Primidone & $42(5.5 \%)$ \\
\hline Zonisamide & $33(4.3 \%)$ \\
\hline Gabapentin & $28(3.7 \%)$ \\
\hline Seizure frequency & $3.4 \pm 3.2$ \\
\hline Disease duration (years) & $17.71 \pm 4.56$ \\
\hline \multicolumn{2}{|l|}{ QOLIE-31score } \\
\hline Seizure concerns & $77.16 \pm 35.05$ \\
\hline Cognitive function & $66.45 \pm 42.93$ \\
\hline Energy/fatigue & $59.33 \pm 18.63$ \\
\hline Emotional wellbeing & $61.27 \pm 18.92$ \\
\hline Social function & $78.00 \pm 21.61$ \\
\hline Medication efficacy & $78.72 \pm 24.12$ \\
\hline Overall quality of life & $61.77 \pm 19.99$ \\
\hline Overall score & $67.81 \pm 20.50$ \\
\hline LSSS score & $54.91 \pm 23.46$ \\
\hline MARS-5 score & $13.32 \pm 6.48$ \\
\hline \multicolumn{2}{|l|}{ Serum AED level, $n(\%)$} \\
\hline Below therapeutic range & $373(48.7 \%)$ \\
\hline Within therapeutic range & $295(38.5 \%)$ \\
\hline Above therapeutic range & $98(12.8 \%)$ \\
\hline
\end{tabular}

QOLIE-31 - Quality of Life in Epilepsy Inventory-31; LSSS - Liverpool Seizure Severity Scale; MARS-5 - Medication Adherence Report Scale. The data is expressed as mean \pm SD unless specifically indicated. Seizure frequency was defined as the mean frequency of complex partial seizures per month during six months of follow-up 
Table 2. The association between MARS-5 score and LSSS score as well as serum AED level

$\begin{array}{lcccc} & \text { B } & \text { SE } & \boldsymbol{\beta} & p^{*} \\ \text { LSSS score } & -0.09 & 0.009 & -0.33 & <0.0001 \\ \text { Serum AED level } & 2.68 & 0.302 & 0.29 & <0.0001\end{array}$

AED - antiepileptic drug; LSSS - Liverpool Seizure Severity Scale; MARS-5 - Medication Adherence Report Scale; SE - standard error. The results were analysed by the multiple linear regression method after adjusting for age, sex, marital status, educational years, employment, income, epilepsy type and disease duration; ${ }^{*} p<0.05$ was considered as statistically significant

$p<0.001)$ showed indirect effects on QoL score. This means that the MARS-5 score can mediate the relationship between LSSS and QOLIE-31, as well as the relationship between AED level and QOLIE-31 (Fig. 1). However, LSSS score and serum AED level did not directly affect the QoL.

\section{Discussion}

This study examined the associations among several variables that influence QoL in the elderly with epilepsy. We suggested a model for the effect of MARS- 5 not only on the relation between seizure severity and QoL, but also on the relation between AED level and QoL. Our findings revealed that serum AED level, medication adherence, and QoL positively correlate with each other in elderly patients with epilepsy. Furthermore, we also found that increased seizure severity, as determined by LSSS score, was associated with decreased medication adherence among elderly patients with epilepsy.

The positive correlation between medication adherence and AED level suggests that the self-reported MARS-5 score could be suitable to assess AED adherence in the elderly with epilepsy, which is consistent with previous studies on

Table 3. Results from multiple linear regression analysis that evaluated the association between dependent (QOLIE-31 score and its domains) and independent (LSSS score, MARS-5 score and serum AED level) variables

\begin{tabular}{|c|c|c|c|c|c|c|}
\hline \multirow[t]{2}{*}{ Variables } & \multicolumn{2}{|c|}{ LSSS } & \multicolumn{2}{|c|}{ serum AED level } & \multicolumn{2}{|c|}{ MARS-5 } \\
\hline & $B(S E)$ & $\beta$ & $\mathrm{B}(\mathrm{SE})$ & $\beta$ & $\mathrm{B}(\mathrm{SE})$ & $\beta$ \\
\hline Seizure concern & $0.07(0.06)$ & 0.05 & $-1.33(1.84)$ & -0.03 & $1.37(0.21)$ & $0.25^{*}$ \\
\hline Cognitive function & $-0.05(0.07)$ & -0.03 & $4.61(2.29)$ & 0.07 & $1.58(0.26)$ & $0.24^{*}$ \\
\hline Energy/fatigue & $-0.07(0.03)$ & -0.08 & $0.57(0.98)$ & 0.02 & $0.57(0.11)$ & $0.20^{*}$ \\
\hline Emotional wellbeing & $-0.04(0.03)$ & -0.06 & $-0.90(1.01)$ & -0.03 & $0.57(0.12)$ & $0.20^{*}$ \\
\hline Social function & $-0.05(0.03)$ & -0.05 & $-0.06(1.16)$ & -0.01 & $0.75(0.13)$ & $0.23^{*}$ \\
\hline Medication efficacy & $-0.05(0.04)$ & -0.05 & $0.91(1.28)$ & 0.03 & $0.64(0.15)$ & $0.17^{*}$ \\
\hline Overall QoL & $-0.01(0.03)$ & -0.02 & $0.70(1.05)$ & 0.02 & $0.46(0.12)$ & $0.15^{*}$ \\
\hline Overall score & $-0.04(0.03)$ & -0.04 & $1.19(1.05)$ & 0.04 & $0.93(0.12)$ & $0.30^{*}$ \\
\hline
\end{tabular}

QOLIE-31 - Quality of Life in Epilepsy Inventory-31; LSSS - Liverpool Seizure Severity Scale; MARS-5 - Medication Adherence Report Scale; AED - antiepileptic drug; QoL - quality of life; SE - standard error. The results were analysed by the Multiple Linear Regression method adjusted for age, sex, marital status, educational years, employment, income, epilepsy type and disease duration. ${ }^{*} p<0.00625$ (Bonferroni correction with eight comparisons; $0.05 / 8=0.00625$ ) was considered as statistically significant

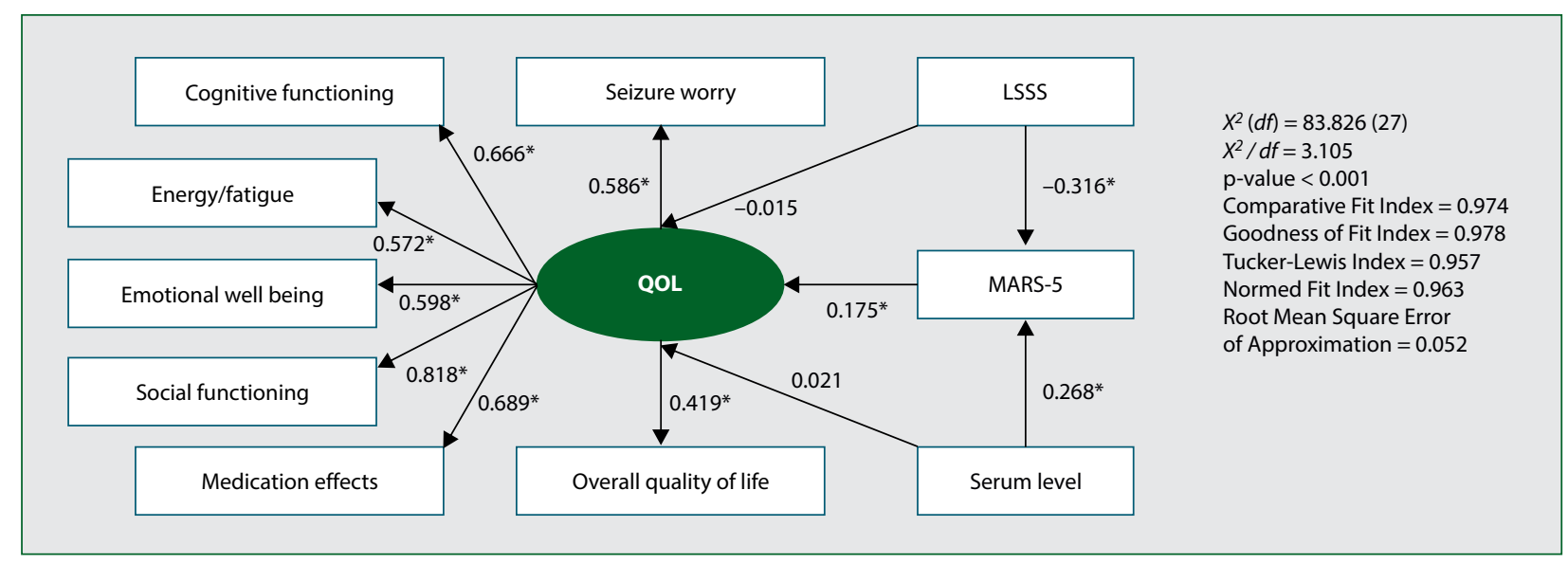

Figure 1. The proxy effects of MARS-5 score on the quality of life (QoL) in elderly patients with epilepsy. ${ }^{*} p<0.001$; LSSS - Liverpool Seizure Severity Scale; MARS-5 - Medication Adherence Report Scale; QOL - quality of life 
epilepsy treatment [25,37]. Given that serum level measurement is invasive and costly, a MARS questionnaire could be used to evaluate patient adherence to treatment. However, in contrast to our findings, several studies on patients with chronic obstructive pulmonary disease (COPD) and children with asthma have suggested that the MARS-5 score is not an accurate self-reporting instrument to measure drug adherence in those patient cohorts $[15,16]$. Furthermore, it has been reported that MARS- 5 score is not an accurate instrument to measure drug adherence in hypertensive patients [14]. The contradictions observed between our results and those in the abovementioned studies might be due to the type of disease and the difference in clinical characteristics, as well as to the efficacy of the medication in controlling medical symptoms.

Our study also indicated that medication adherence was inversely correlated with seizure severity. However, this finding could have been confounded by several clinical and demographic characteristics of the participants. Therefore, we adjusted our analyses for confounders such as age, sex, marital status, educational years, employment status, income level, epilepsy type, and duration of the disease. Interestingly, we observed that seizure severity and serum drug level were both independently related to medication adherence even after adjusting for those confounders. Therefore, our findings show that seizure severity and serum AED level are strong predictors of medication adherence in patients with epilepsy.

In this study, we evaluated the association between medication adherence, seizure severity and serum AED level of the patients with their QoL. In particular, we found that, after controlling clinical and demographic characteristics, medication adherence was directly associated with the overall score of QoL and all its domains, while seizure severity and serum AED level did not have a significant correlation with the QoL. Serum AED levels are not necessarily associated with QoL in patients with epilepsy. That being said it does not provide detailed information on patients' level of adherence over time. Moreover, serum AED levels can only be assessed in patients who are taking second generation AEDs [40]. Moreover, the interpretation of serum AED level depends on the time of sampling and the duration of AED therapy. These problems limit the usefulness of the information regarding serum AED levels in everyday clinical practice.

Although our findings did not confirm a significant correlation between seizure severity and QoL, several studies have confirmed an inverse relationship between seizure severity and QoL in epileptic patients. For example, Harden et al. [41] examined a group of women aged 18-45 with refractory epilepsy and found that, even when controlling for depression, seizure severity was inversely correlated with multiple domains of QoL. Bautista et al. [42] indicated that quality of life for patients with epilepsy was adversely affected by seizure severity. Furthermore, Sancho et al. [43] indicated that QoL in patients with severe seizures has been consistently shown to be worse than for those with mild or moderate seizures.
Moreover, our study indicated that medication adherence was positively correlated with QoL. Indeed, AED non-adherence impacted negatively on QoL as a result of poor seizure control. A number of studies have examined the relationship between medication adherence and QoL. However, the findings remain controversial. Consistent with our findings, several studies have previously demonstrated the beneficial effects of medication adherence on improved QoL in epileptic patients $[10,44,45]$ and other diseases [46, 47]. Loon et al. [47] found that adherence to glaucoma medications was associated with better QoL. Moreover, medication adherence has been reported to be associated with increased QoL in patients with epilepsy [44, 45]. However, Martinez et al. [48] could not find any significant association between medication adherence and QoL in patients with type 2 diabetes. In the study by Saleem et al. [21], no relationship was found between medication adherence and QoL in hypertensive patients. However, due to the conflicting results, it remains unclear whether increased seizure severity or decreased QoL is the primary event.

Despite the evidence that medication adherence can improve QoL, it is uncertain whether seizure severity and serum AED level can be replaced by medication adherence to predict QoL. Our hypothesis was that medication adherence may mediate the correlation between seizure severity and QoL, as well as serum AED level and QoL. Using the Sobel test to test our hypothesis revealed that medication adherence did mediate the correlation between seizure severity and QoL. This indicates that less severe seizures may be due to increased medication adherence and eventually lead to increased QoL. Moreover, the Sobel test revealed the mediatory effect of medication adherence on AED level and QoL, suggesting that a higher AED level can be due to increased medication adherence resulting in improved QoL. In addition, these results were supported by the SEM model, which confirmed the proxy effect of medication adherence on the latent score of QoL.

This study revealed that neither seizure severity nor serum AED level had a direct effect on the QoL, although there was an indirect effect between these variables via medication adherence. Therefore, medication adherence is very important to improve QoL in elderly patients with epilepsy, and it should be strongly encouraged by physicians.

According to our findings, elderly people with epilepsy who are concerned about the impact of epilepsy on their independence and QoL could potentially control their seizure symptoms through medication adherence. Furthermore, there is a need to pay more attention to medication management and adherence to improve QoL among the elderly with epilepsy. As the elderly are prone to multiple comorbidities, they are at higher risk of polypharmacy, and therefore may present with a higher risk of non-adherence to medication compared to the younger population. This results in decreased therapeutic benefits for the patient, and increased healthcare expenditure.

There are several strengths of our study, including serum AED level measurement to assess medication adherence, 
a relatively large sample size, and longitudinal design, which can help yield firm conclusions. However, some caution is necessary in the interpretation of our findings. Firstly, it is likely that the associations between the variables may also be partially explained by other confounders that were not controlled for in this study, e.g. unhealthy lifestyle. Secondly, we did not assess serum AED level and the QoL at the beginning of the study. Therefore, we are unable to discuss the association between the changes of medical adherence and quality of life throughout the study. Finally, major cognitive and acute psychiatric disorders such as depression and anxiety, which could be key factors affecting both medication adherence and QoL, were excluded from the current study.

\section{Conclusion}

In elderly patients with epilepsy, medication adherence significantly correlates with QoL. Given proper medication management, the QoL of these patients can be improved. Therefore, healthcare providers need to provide an appropriate level of support by frequently reviewing drug management and monitoring adherence in elderly patients with epilepsy.

\section{References}

1. Ngugi AK, Bottomley C, Kleinschmidt I, et al. Estimation of the burden of active and life-time epilepsy: a meta-analytic approach. Epilepsia. 2010; 51(5): 883-890, doi: 10.1111/j.1528-1167.2009.02481.x, indexed in Pubmed: 20067507.

2. Jacoby A. Stigma, epilepsy, and quality of life. Epilepsy \& Behavior. 2002; 3(6): 10-20, doi: 10.1016/s1525-5050(02)00545-0.

3. Epilepsy. http://www.who.int/mediacentre/factsheets/fs999/en/ (Accessed Nov 17, 2017).

4. Mohammadi M, Ghanizadeh A, Davidian H, et al. Prevalence of epilepsy and comorbidity of psychiatric disorders in Iran. Seizure. 2006; 15(7): 476-482, doi: 10.1016/j.seizure.2006.05.011.

5. Ebrahimi H, Shafa M, Hakimzadeh AsI S. Prevalence of active epilepsy in Kerman, Iran: a house based survey. Acta Neurol Taiwan. 2012; 21(3): 115-124, indexed in Pubmed: 23196731.

6. Helmers SL, Thurman DJ, Durgin TL, et al. Descriptive epidemiology of epilepsy in the U.S. population: A different approach. Epilepsia. 2015; 56(6): 942-948, doi: 10.1111/epi.13001, indexed in Pubmed: 25921003.

7. Brodie MJ, Elder AT, Kwan P. Epilepsy in later life. Lancet Neurol. 2009; 8(11): 1019-1030, doi: 10.1016/S1474-4422(09)70240-6, indexed in Pubmed: 19800848.

8. Chen LA, Cheng SJ, Jou SB. Epilepsy in the Elderly. International Journal of Gerontology. 2012; 6(2): 63-67, doi: 10.1016/j.jige.2012.05.013.

9. Ageing W. http://www. who. int/mediacentre/factsheets/fs404/en/ (Accessed September 2015).

10. Eatock J, Baker GA. Managing patient adherence and quality of life in epilepsy. Neuropsychiatr Dis Treat. 2007; 3(1): 117-131, indexed in Pubmed: 19300542.

11. Sabaté E. Adherence to long-term therapies: evidence for action: Genava: World Health Organization. ; 2003.
12. Stephen $\sqcup$, Brodie MJ. Epilepsy in elderly people. Lancet. 2000; 355(9213): 1441-1446, doi: 10.1016/S0140-6736(00)02149-8, indexed in Pubmed: 10791538.

13. Sander JW. The use of antiepileptic drugs--principles and practice. Epilepsia. 2004; 45 Suppl 6: 28-34, doi: 10.1111/j.0013-9580.2004.455005.x, indexed in Pubmed: 15315513.

14. van de Steeg N, Sielk M, Pentzek M, et al. Drug-adherence questionnaires not valid for patients taking blood-pressure-lowering drugs in a primary health care setting. J Eval Clin Pract. 2009; 15(3): 468472, doi: 10.1111/j.1365-2753.2008.01038.x, indexed in Pubmed: 19366389.

15. Tommelein E, Mehuys E, Van Tongelen I, et al. Accuracy of the Medication Adherence Report Scale (MARS-5) as a quantitative measure of adherence to inhalation medication in patients with COPD. Ann Pharmacother. 2014; 48(5): 589-595, doi: 10.1177/1060028014522982, indexed in Pubmed: 24523393.

16. Garcia-Marcos PW, Brand PLP, Kaptein AA, et al. Is the MARS questionnaire a reliable measure of medication adherence in childhood asthma? J Asthma. 2016; 53(10): 1085-1089, doi: 10.1080/02770903.2016.1180699, indexed in Pubmed: 27177241.

17. Hansen R, Seifeldin R, Noe L. Medication adherence in chronic disease: issues in posttransplant immunosuppression. Transplant Proc. 2007; 39(5): 1287-1300, doi: 10.1016/j.transproceed.2007.02.074, indexed in Pubmed: 17580126.

18. Khanna R, Pace PF, Mahabaleshwarkar R, et al. Medication adherence among recipients with chronic diseases enrolled in a state Medicaid program. Popul Health Manag. 2012; 15(5): 253-260, doi: 10.1089/pop.2011.0069, indexed in Pubmed: 22401152.

19. Asadi-Pooya AA. Drug compliance of children and adolescents with epilepsy. Seizure. 2005; 14(6): 393-395, doi: 10.1016/j.seizure.2005.05.003, indexed in Pubmed: 15978849.

20. Jones RM, Butler JA, Thomas VA, et al. Adherence to treatment in patients with epilepsy: associations with seizure control and illness beliefs. Seizure. 2006; 15(7): 504-508, doi: 10.1016/j.seizure.2006.06.003, indexed in Pubmed: 16861012.

21. Saleem F, Hassali MA, Shafie AA, et al. Does treatment adherence correlates with health related quality of life? Findings from a cross sectional study. BMC Public Health. 2012; 12: 318, doi: 10.1186/14712458-12-318, indexed in Pubmed: 22545950.

22. Liu J, Liu Z, Ding Hu, et al. Adherence to treatment and influencing factors in a sample of Chinese epilepsy patients. Epileptic Disord. 2013; 15(3): 289-294, doi: 10.1684/epd.2013.0588, indexed in Pubmed: 23906632.

23. Getnet A, Woldeyohannes SM, Bekana L, et al. Antiepileptic Drug Nonadherence and Its Predictors among People with Epilepsy. Behav Neurol. 2016; 2016: 3189108, doi: 10.1155/2016/3189108, indexed in Pubmed: 28053370.

24. Osterberg L, Blaschke T. Adherence to medication. N Engl J Med. 2005; 353(5): 487-497, doi: 10.1056/NEJMra050100, indexed in Pubmed: 16079372.

25. Jimmy B, Jose J. Patient medication adherence: measures in daily practice. Oman Med J. 2011; 26(3): 155-159, doi: 10.5001/ omj.2011.38, indexed in Pubmed: 22043406.

26. Pakpour AH, Gholami M, Esmaeili R, et al. A randomized controlled multimodal behavioral intervention trial for improving antiepileptic drug adherence. Epilepsy Behav. 2015; 52(Pt A): 133-142, doi: 10.1016/j.yebeh.2015.08.036, indexed in Pubmed: 26414343. 
27. Gaitatzis A, Sander JW, Sander JW. The epidemiology of epilepsy revisited. Curr Opin Neurol. 2003; 16(2): 165-170, doi: 10.1097/01. wco.0000063766.15877.8e, indexed in Pubmed: 12644744.

28. Kim S, Bennett $\mathrm{K}$, Wallace $\mathrm{E}$, et al. Measuring medication adherence in older community-dwelling patients with multimorbidity. European Journal of Clinical Pharmacology. 2017; 74(3): 357-364, doi: 10.1007/s00228-017-2388-y.

29. Ahmad N, Othaman NI, Islahudin F. Medication adherence and quality of life in epilepsy patients. Int J Pharm PharmSci. 2013; 5: 401-4.

30. Lin CY, Chen H, Pakpour AH. Correlation between adherence to antiepileptic drugs and quality of life in patients with epilepsy: A longitudinal study. Epilepsy Behav. 2016; 63: 103-108, doi: 10.1016/j. yebeh.2016.07.042, indexed in Pubmed: 27588360.

31. Meador KJ, Gilliam FG, Kanner AM, et al. Cognitive and behavioral effects of antiepileptic drugs. Epilepsy Behav. 2001; 2(4): SS1-SS17, doi: 10.1006/ebeh.2001.0235, indexed in Pubmed: 12609218.

32. Berg AT, Berkovic SF, Brodie MJ, et al. Revised terminology and concepts for organization of seizures and epilepsies: report of the ILAE Commission on Classification and Terminology, 2005-2009. Epilepsia. 2010; 51(4): 676-685, doi: 10.1111/j.1528-1167.2010.02522.x, indexed in Pubmed: 20196795.

33. Horne R, Weinman J. Self-regulation and Self-management in Asthma: Exploring The Role of Illness Perceptions and Treatment Beliefs in Explaining Non-adherence to Preventer Medication. Psychology \& Health. 2002; 17(1): 17-32, doi: 10.1080/08870440290001502.

34. Jónsdóttir H, Opjordsmoen S, Birkenaes AB, et al. Medication adherence in outpatients with severe mental disorders: relation between self-reports and serum level. J Clin Psychopharmacol. 2010; 30(2): 169-175, doi: 10.1097/JCP.0b013e3181d2191e, indexed in Pubmed: 20520290.

35. Baker GA, Smith DF, Jacoby A, et al. Liverpool Seizure Severity Scale revisited. Seizure. 1998; 7(3): 201-205, indexed in Pubmed: 9700832.

36. Affolter N, Krähenbühl S, Schlienger RG. Appropriateness of serum level determinations of antiepileptic drugs. Swiss Med Wkly. 2003; 133(43-44): 591-597, doi: 2003/43/smw-10435, indexed in Pubmed: 14745654.

37. Cramer JA, Perrine K, Devinsky O, et al. Development and cross-cultural translations of a 31-item quality of life in epilepsy inventory. Epilepsia. 1998; 39(1): 81-88, indexed in Pubmed: 9578017.

38. Mohammadi N, Kian S, Davoudi F, et al. Psychometric evaluation of the Persian version of the quality of life in epilepsy inventory-31. Iran J Neurol. 2013; 12(4): 144-148, indexed in Pubmed: 24250924.
39. Hu L, Bentler P. Cutoff criteria for fit indexes in covariance structure analysis: Conventional criteria versus new alternatives. Structural Equation Modeling: A Multidisciplinary Journal. 1999; 6(1): 1-55, doi: 10.1080/10705519909540118.

40. Walters RJL, Hutchings AD, Smith DF, et al. Inappropriate requests for serum anti-epileptic drug levels in hospital practice. QJM. 2004; 97(6): 337-341, indexed in Pubmed: 15152107.

41. Harden CL, Maroof DA, Nikolov B, et al. The effect of seizure severity on quality of life in epilepsy. Epilepsy Behav. 2007; 11(2): 208-211, doi: 10.1016/j.yebeh.2007.05.002, indexed in Pubmed: 17604229.

42. Bautista RE, Glen ET. Seizure severity is associated with quality of life independent of seizure frequency. Epilepsy Behav. 2009; 16(2): 325-329, doi: 10.1016/j.yebeh.2009.07.037, indexed in Pubmed: 19713158.

43. Sancho J, Iváñez V, Molins A, et al. Changes in seizure severity and quality of life in patients with refractory partial epilepsy. Epilepsy Behav. 2010; 19(3): 409-413, doi: 10.1016/j.yebeh.2010.08.011, indexed in Pubmed: 20855235.

44. Hovinga CA, Asato MR, Manjunath R, et al. Association of non-adherence to antiepileptic drugs and seizures, quality of life, and productivity: survey of patients with epilepsy and physicians. Epilepsy Behav. 2008; 13(2): 316-322, doi: 10.1016/j.yebeh.2008.03.009, indexed in Pubmed: 18472303.

45. Martins H, Alonso N, Guilhoto L, et al. Adherence to treatment in patients with juvenile myoclonic epilepsy: correlation with quality of life and adverse effects of medication. Journal of Epilepsy and Clinical Neurophysiology. 2009; 15(4): 192-196, doi: 10.1590/s1676 26492009000400010.

46. Alfian SD, Sukandar H, Lestari K, et al. Medication Adherence Contributes to an Improved Quality of Life in Type 2 Diabetes Mellitus Patients: A Cross-Sectional Study. Diabetes Ther. 2016; 7(4): 755-764, doi: 10.1007/s13300-016-0203-x, indexed in Pubmed: 27718218.

47. Loon SC, Jin J, Jin Goh M. The relationship between quality of life and adherence to medication in glaucoma patients in Singapore. J Glaucoma. 2015; 24(5): e36-e42, doi: 10.1097/JJG.00000000000000007, indexed in Pubmed: 24248002.

48. Martínez YV, Prado-Aguilar CA, Rascón-Pacheco RA, et al. Quality of life associated with treatment adherence in patients with type 2 diabetes: a cross-sectional study. BMC Health Serv Res. 2008; 8: 164, doi: 10.1186/1472-6963-8-164, indexed in Pubmed: 18667076. 